\title{
A note on a result of Bateman and Chowla
}

\author{
by \\ P. CodecÀ (Ferrara) and M. NAIR (Glasgow)
}

1. Introduction. In 1961, answering a problem proposed by N. J. Fine, Besicovitch [2] constructed an example of a non-trivial real continuous function $f$ on $[0,1]$ which is not odd with respect to the point $1 / 2$ and with the property that

$$
\sum_{a=1}^{n} f\left(\frac{a}{n}\right)=0 \quad \text { for each } n \in \mathbb{N} .
$$

His proof consisted in the definition of the required function in inductive stages on small subintervals of $[0,1]$ and, in modern terminology, is rather akin to the construction of a complicated fractal function.

Bateman and Chowla [1], in 1963, pointed out that the more explicit functions

$$
f_{1}(\theta)=\sum_{n=1}^{\infty} \frac{\lambda(n)}{n} \cos 2 \pi n \theta
$$

where $\lambda$ denotes the Liouville function and

$$
f_{2}(\theta)=\sum_{n=1}^{\infty} \frac{\mu(n)}{n} \cos 2 \pi n \theta
$$

where $\mu$ denotes the Möbius function also share the above properties of Besicovitch's function. The continuity of these two functions follows from the uniform convergence of the series involved, which is a classical result of Davenport [3]. The other properties including (1) are then comparatively trivial to demonstrate.

From a heuristic point of view, it is by no means clear from their paper why one might expect, a priori, functions such as (2) or (3) to be associated with Fine's problem.

In this paper, we show that a class of functions, which includes Davenport's function (3), arises naturally as formal infinite limits of a finite

2000 Mathematics Subject Classification: Primary 11N99. 
minimizing problem involving sums of type (1). We then show that each member of this class provides in fact a solution to Fine's problem. To do this, we prove a Davenport-type uniform convergence result of the series involved using Vaughan's identity, and one interesting outcome of our work is that the function

$$
f(\theta)=\sum_{n=1}^{\infty} \frac{\mu(n)}{\sigma(n)} \cos 2 \pi n \theta,
$$

where $\sigma$ is the usual sum of divisors function, is in a sense a more natural solution to the original problem than is (3). Our main result is thus the following.

THEOREM 1. Let $h(n)$ be any positive multiplicative function with

$$
h(p)=1+O\left(\frac{\log p}{p}\right) \quad \text { for primes } p .
$$

Then $f(x)$ defined by

$$
f(x)=\sum_{k=1}^{\infty} h(k) \frac{\mu(k)}{k} \cos 2 \pi k x
$$

is a non-trivial function, continuous on $[0,1]$, which satisfies $f(x)=f(1-x)$ and has the property that

$$
\sum_{a=1}^{n} f(a / n)=0 \quad \text { for each } n \in \mathbb{N} .
$$

2. A finite minimizing problem and its solution. For any real function $f$, continuous on $[0,1]$, define its deviation $D(n)=D_{f}(n)$, of order $n$, by

$$
D(n)=\frac{1}{n} \sum_{a=1}^{n} f\left(\frac{a}{n}\right)-\int_{0}^{1} f(x) d x \quad \text { for any } n \in \mathbb{N} .
$$

Clearly $D_{f}(n)=D_{g}(n)$ if $f$ and $g$ differ by a constant. For even trigonometric polynomials

$$
f(x)=f_{N}(x)=\sum_{k \leq N} \frac{c(k)}{k} \cos 2 \pi k x
$$

we see that, for any fixed $N \in \mathbb{N}$ and $c(k) \in \mathbb{R}$,

$$
D(n)=\frac{1}{n} \sum_{a=1}^{n} f\left(\frac{a}{n}\right)=\sum_{\substack{k \leq N \\ n \mid k}} \frac{c(k)}{k} .
$$


We consider the problem of determining a function of the form (5) which minimizes the weighted $l^{2}$-norm $\left\|D_{N}\right\|$ of the deviations $D(n)$ defined by

$$
\left\|D_{N}\right\|^{2}=\sum_{n \leq N} \alpha_{n} D^{2}(n)=\sum_{n \leq N} \frac{\alpha_{n}}{n^{2}}\left(\sum_{h \leq N / n} \frac{c(h n)}{h}\right)^{2}
$$

subject to the normalizing condition $c(1)=1$ and where $\alpha_{n}$ are any given positive numbers.

Theorem 2. For $N \in \mathbb{N}$ define a class $S_{N}$ of real trigonometric polynomials of order $N$ by

$$
S_{N}=\left\{f: f(x)=\sum_{k \leq N} \frac{c(k)}{k} \cos 2 \pi k x, c(k) \in \mathbb{R}, c(1)=1\right\} .
$$

Then for any real positive $\alpha_{n}$ and any $f \in S_{N}$,

$$
\left\|D_{N}\right\|^{2} \geq \frac{1}{\sum_{n \leq N} \mu^{2}(n) / \alpha_{n}}
$$

with equality for the polynomial $f \in S_{N}$ with

$$
\frac{c(k)}{k}=\frac{1}{\sum_{n \leq N} \mu^{2}(n) / \alpha_{n}}\left(\sum_{\substack{n \leq N / k \\(n, k)=1}} \frac{\mu^{2}(n)}{\alpha_{n k}}\right) \mu(k) \quad \text { for each } k, 1 \leq k \leq N .
$$

Pr o of. The condition $c(1)=1$ can be expressed as

$$
\sum_{\substack{h, n \\ h n \leq N}} \frac{c(h n) \mu(n)}{h n}=\sum_{l \leq N} \frac{c(l)}{l} \sum_{n \mid l} \mu(n)=1
$$

and hence

$$
\sum_{n \leq N} \frac{\mu(n)}{\alpha_{n}^{1 / 2}} \cdot \frac{\alpha_{n}^{1 / 2}}{n} \sum_{h \leq N / n} \frac{c(h n)}{h}=1
$$

We apply the Cauchy-Schwarz inequality to this condition in a manner reminiscent of Turán's proof of Selberg's Upper Bound Sieve (see HalberstamRichert [4], p. 121) to obtain

$$
\sum_{n \leq N} \frac{\mu^{2}(n)}{\alpha_{n}} \sum_{n \leq N} \frac{\alpha_{n}}{n^{2}}\left(\sum_{h \leq N / n} \frac{c(h n)}{h}\right)^{2} \geq 1,
$$

i.e. that

$$
\left\|D_{N}\right\|^{2} \geq \frac{1}{\sum_{n \leq N} \mu^{2}(n) / \alpha_{n}}
$$


with equality when

$$
\frac{\mu(n)}{\alpha_{n}^{1 / 2}}=C \frac{\alpha_{n}^{1 / 2}}{n} \sum_{h \leq N / n} \frac{c(h n)}{h}
$$

for some $C \neq 0$ and all $n \leq N$. By Möbius inversion,

$$
\frac{c(k)}{k}=\frac{1}{C} \sum_{h \leq N / k} \frac{\mu(h) \mu(h k)}{\alpha_{h k}}=\frac{1}{C}\left(\sum_{\substack{h \leq N / k \\(h, k)=1}} \frac{\mu^{2}(h)}{\alpha_{h k}}\right) \mu(k) .
$$

The condition $c(1)=1$ forces the choice $C=\sum_{h \leq N} \mu^{2}(h) / \alpha_{h}$, and this completes the proof of Theorem 2 .

Now suppose that the positive weights $\alpha_{n}$ are multiplicative functions of $n$ with

$$
\alpha_{p}=1+O\left(\frac{\log p}{p}\right)
$$

We shall determine the formal limit of the minimizing polynomial in Theorem 2 as $N \rightarrow \infty$ by calculating the limit of $c(k) / k$ as $N \rightarrow \infty$ for each fixed $k$. Clearly

$$
\frac{c(k)}{k}=\left(\frac{\sum_{n \leq N / k,(n, k)=1} \mu^{2}(n) / \alpha_{n}}{\sum_{n \leq N} \mu^{2}(n) / \alpha_{n}}\right) \frac{\mu(k)}{\alpha_{k}} .
$$

Writing $\beta(n)=1 / \alpha_{n}$, we have for $\operatorname{Re} s>1$,

$$
\begin{aligned}
\sum_{\substack{n=1 \\
(n, k)=1}}^{\infty} \frac{\mu^{2}(n) \beta(n)}{n^{s}} & =\prod_{p}\left(1+\frac{\beta(p)}{p^{s}}\right) \prod_{p \mid k}\left(1+\frac{\beta(p)}{p^{s}}\right)^{-1} \\
& =F(s) G(s, k), \quad \text { say. }
\end{aligned}
$$

Writing $\beta(p)=1+R(p)$, where by hypothesis $R(p)=O((\log p) / p)$, we obtain

$$
F(s)=\frac{\zeta(s)}{\zeta(2 s)} \prod_{p}\left(1+\frac{R(p)}{p^{s}+1}\right)
$$

and hence $F(s)$ is analytic in a region which includes $\operatorname{Re} s \geq 1$ except for a simple pole at $s=1$ with residue

$$
\frac{1}{\zeta(2)} \prod_{p}\left(1+\frac{R(p)}{p+1}\right) .
$$

Therefore by the Wiener-Ikehara Theorem, or indeed by more elementary 
means, it follows from (9) that

$$
\lim _{x \rightarrow \infty} \frac{1}{x} \sum_{\substack{n \leq x \\(n, k)=1}} \mu^{2}(n) \beta(n)=\frac{1}{\zeta(2)} \prod_{p}\left(1+\frac{R(p)}{p+1}\right) \prod_{p \mid k}\left(1+\frac{\beta(p)}{p}\right)^{-1} .
$$

A simple calculation then yields that, for fixed $k \in \mathbb{N}$,

$$
\lim _{N \rightarrow \infty} c(k)=\prod_{p \mid k}\left(\alpha_{p}+\frac{1}{p}\right)^{-1} \mu(k) .
$$

Hence the formal limit of the minimizing polynomial is given by

$$
f(x)=\sum_{k=1}^{\infty} \prod_{p \mid k}\left(\alpha_{p}+\frac{1}{p}\right)^{-1} \frac{\mu(k)}{k} \cos 2 \pi k x .
$$

Note that the choice $\alpha_{p}=1-1 / p$, i.e. $\alpha_{k}=\phi(k) / k$, yields Davenport's function (3) whilst the equal weights $\alpha_{k}=1$ give the function (4) mentioned in the introduction.

REMARK. Although the condition (8) on $\alpha_{p}$ is principally chosen here to facilitate calculations in the application of Vaughan's identity, in particular it ensures that $\alpha_{p}$ are not too small and hence the function

$$
h(n)=\prod_{p \mid n}\left(\alpha_{p}+\frac{1}{p}\right)^{-1}
$$

satisfies $h(n) \ll(\log n)^{c}$ for some $c>0$; it is equally true that $\alpha_{p}$ cannot be too large since we can show that $\sum_{n=1}^{\infty} h(n) / n$ needs to be necessarily divergent for the overall function $f(x)$ to have all the desired properties.

3. Proof of Theorem 1. Our Theorem 3 proved below implies that

$$
\sum_{k \leq y} \mu(k) h(k) \cos 2 \pi k x \ll y / \log ^{\lambda} y
$$

uniformly in $x$, for any $\lambda>0$. Writing

$$
S_{N}(x)=\sum_{k \leq N} \frac{\mu(k) h(k)}{k} \cos 2 \pi k x,
$$

we deduce, by partial summation, that

$$
\begin{aligned}
S_{N+M}(x) & -S_{N}(x) \\
= & \left.\sum_{k \leq N+M} \mu(k) h(k) \cos 2 \pi k x\right) \frac{1}{N+M} \\
& -\left(\sum_{k \leq N} \mu(k) h(k) \cos 2 \pi k x\right) \frac{1}{N}+\int_{N}^{N+M}\left(\sum_{k \leq t} \mu(k) h(k) \cos 2 \pi k x\right) \frac{d t}{t^{2}} .
\end{aligned}
$$


This implies, using Theorem 3 with $\lambda>1$, that $S_{N}(x)$ converges uniformly in $x$ and hence that $f(x)$ given by (10) is continuous. Integrating the series term by term, we deduce that

$$
\int_{0}^{1} f(x) d x=0
$$

and, by Parseval's identity,

$$
\int_{0}^{1} f^{2}(x) d x=\frac{1}{2} \sum_{k=1}^{\infty} \frac{\mu^{2}(k) h^{2}(k)}{k^{2}} \geq \frac{1}{2}
$$

so that $f(x)$ is non-trivial. In addition, setting $g(k)=\mu(k) h(k)$, we find that for any $n \in \mathbb{N}$,

$$
\begin{aligned}
\sum_{a=1}^{n} f\left(\frac{a}{n}\right) & =\sum_{a=1}^{n}\left(\sum_{k=1}^{\infty} \frac{g(k)}{k} \cos \frac{2 \pi k a}{n}\right) \\
& =\sum_{k=1}^{\infty} \frac{g(k)}{k} \sum_{a=1}^{n} \cos \frac{2 \pi k a}{n}=n \sum_{\substack{k=1 \\
n \mid k}}^{\infty} \frac{g(k)}{k}=\left(\sum_{\substack{h=1 \\
(h, n)=1}}^{\infty} \frac{g(h)}{h}\right) g(n) .
\end{aligned}
$$

Now for $\operatorname{Re} s>1$, observe that

$$
\sum_{\substack{h=1 \\(h, n)=1}}^{\infty} \frac{g(h)}{h^{s}}=\prod_{p \nmid n}\left(1+\frac{g(p)}{p^{s}}\right)=\frac{1}{\zeta(s)} G(s)
$$

where $G(s)$ is analytic in a region which contains the point $s=1$. Hence by the continuity theorem for Dirichlet series, we see that for all $n \in \mathbb{N}$,

$$
\sum_{\substack{h=1 \\(h, n)=1}}^{\infty} \frac{g(h)}{h}=\lim _{s \rightarrow 1} \frac{G(s)}{\zeta(s)}=0,
$$

which implies that $\sum_{a=1}^{n} f(a / n)=0$ for all $n \in \mathbb{N}$, as required.

This completes the proof of Theorem 1. We now prove, as required, Theorem 3.

THEOREM 3. Let $h(n)$ be any positive multiplicative function with

$$
h(p)=1+O\left(\frac{\log p}{p}\right) \quad \text { for primes } p .
$$

Then, for any $\lambda>0$,

$$
\max _{\alpha \in[0,1]}\left|\sum_{n \leq x} \mu(n) h(n) e(n \alpha)\right| \ll_{\lambda} x / \log ^{\lambda} x
$$

where, as usual, $e(n \alpha)=\exp (2 \pi i n \alpha)$ and $\ll_{\lambda}$ indicates the Vinogradov symbol with the implicit constant depending at most on $\lambda$. 
REMARK. With a more judicious choice of the parameters involved, it is easily seen that the hypothesis on $h$ can be relaxed to

$$
h(p)=1+O\left(1 / p^{1 / 2}\right)
$$

and the bound obtained can be sharpened to

$$
\ll x \exp \left(-c_{0}(\log x)^{1 / 2}\right) .
$$

We have refrained from doing this since we only need Theorem 3 as stated and even so in fact only for some $\lambda>1$.

Proof (of Theorem 3). Set $g(n)=\mu(n) h(n)$ and note that $g(n) \ll \log ^{c} n$ for some fixed $c \geq 1$. We need the following Siegel-Walfisz type result due to Siebert [5], Satz 4.

Lemma 1. Let $f(n)$ be a multiplicative function with

$$
\sum_{p \leq x}|f(p)+\tau| \ll x^{1-\varepsilon}
$$

where $\varepsilon>0, \tau \in \mathbb{N}$ and $\left|f\left(p^{a}\right)\right| \leq c_{1} a^{c_{2}}$ with $a \in \mathbb{N}$ and $c_{1}, c_{2}>0$. Then for any $h>0$ and $\theta=\theta(h)>0$,

$$
\sum_{\substack{n \leq x \\ n \equiv l(\bmod k)}} f(n) \ll x \exp \left(-\theta(\log x)^{1 / 2}\right)
$$

uniformly for $k \leq \log ^{h} x$.

Observe that $g(n)$ satisfies the hypotheses of Lemma 1 with $\tau=1$. Note also that the upper bound in Theorem 3 for $\alpha=0$ and $\alpha=1$ follows immediately from this lemma so that we may assume henceforth that $\alpha \in$ $(0,1)$.

For any $Q \in \mathbb{N}$, Dirichlet's theorem implies that there exist $a, q \in \mathbb{N}$ with $(a, q)=1$ and $q \leq Q$ such that

$$
\left|\alpha-\frac{a}{q}\right| \leq \frac{1}{q Q} .
$$

Put $Q=x(\log x)^{-\lambda_{1}}, \delta=(\log x)^{\lambda_{1}}$ where $\lambda_{1}$ satisfies

$$
\lambda_{1} \geq 2 \lambda+2 c+5,
$$

$c$ as in the upper bound for $g(n)$. We define the major arcs to consist of those $\alpha$ with corresponding $q \leq \delta$ and the minor arcs those $\alpha$ with $\delta<q \leq Q$.

Write

$$
M_{n}=\sum_{m \leq n} g(m) e(a m / q)
$$


for each $\alpha \in(0,1)$. A simple calculation involving partial summation yields

$$
\left|\sum_{n \leq x} g(n) e(n \alpha)\right| \leq\left(1+\frac{2 \pi x}{q Q}\right) \max _{n \leq x}\left|M_{n}\right| .
$$

On the major arcs, writing

$$
M_{n}=\sum_{r=0}^{q-1} e(a r / q) \sum_{\substack{m \leq n \\ m \equiv r(\bmod q)}} g(m)
$$

and using (11) and Lemma 1, one easily obtains

$$
\begin{aligned}
\left|\sum_{n \leq x} g(n) e(n \alpha)\right| & \leq \max _{n \leq x} \max _{0 \leq r \leq q-1}(q+2 \pi x / Q)\left|\sum_{\substack{m \leq n \\
m \equiv r(\bmod q)}} g(m)\right| \\
& \ll x \exp \left(-\theta\left(\lambda_{1}\right)(\log x)^{1 / 2}\right)(\log x)^{\lambda_{1}} \ll x / \log ^{\lambda} x .
\end{aligned}
$$

On the minor arcs we have $q Q>x$ and hence from (11), it suffices to show that

$$
\max _{n \leq x}\left|M_{n}\right| \ll_{\lambda} x / \log ^{\lambda} x .
$$

Since, trivially, $M_{n} \ll n(\log n)^{c}$, it suffices to prove that

$$
M_{N} \ll x / \log ^{\lambda} x
$$

for any $N$ with $x(\log x)^{-\lambda_{1}} \leq N \leq x$.

Put $u=N^{2 / 5}$. Vaughan's identity [6] yields the decomposition

$$
M_{N}=S_{0}+S_{1}-S_{2}-S_{3}
$$

where

$$
\begin{aligned}
S_{0} & =\sum_{n \leq u} g(n) e(n a / q), \\
S_{1} & =\sum_{d \leq u} \mu(d) \sum_{r \leq N / d} \sum_{n \leq N /(d r)} g(n) e(d r n a / q), \\
S_{2} & =\sum_{d \leq u} \mu(d) \sum_{n \leq u} \sum_{r \leq N /(d n)} g(n) e(d r n a / q), \\
S_{3} & =\sum_{u \leq m \leq N / u} \tau(m) \sum_{u<n \leq N / m} g(n) e(m n a / q) .
\end{aligned}
$$

Trivially, we have, for any $\varepsilon>0$,

$$
S_{0} \ll u(\log x)^{c} \ll x^{2 / 5+\varepsilon} .
$$

To estimate $S_{1}$, writing $r n=k$, we see that

$$
S_{1}=\sum_{d \leq u} \mu(d) \sum_{k \leq N / d} e(d k a / q) \sum_{n \mid k} g(n)
$$


and hence

$$
S_{1} \ll \sum_{d \leq u} \sum_{k \leq N / d}\left|\sum_{n \mid k} g(n)\right| .
$$

Using $\left|\sum_{n \mid k} g(n)\right|=\prod_{p \mid k}|1-h(p)|$, we deduce that

$$
\sum_{k \leq N / d}\left|\sum_{n \mid k} g(n)\right| \leq \sum_{k \leq N / d}\left|\sum_{n \mid k} g(n)\right|\left(\frac{N}{d k}\right)^{1 / 2} \ll\left(\frac{N}{d}\right)^{1 / 2}
$$

and hence

$$
S_{1} \ll N^{1 / 2} u^{1 / 2} \ll x^{7 / 10} .
$$

For the estimation of $S_{2}$ and $S_{3}$, we need Lemma 2.2 of Vaughan [6] which we state here in two parts.

Lemma 2. (i) For $N_{1}, N_{2} \in \mathbb{Z}$ and $N_{2} \geq N_{1}$,

$$
\left|\sum_{n=N_{1}}^{N_{2}} e\left(\frac{n a}{q}\right)\right| \leq \min \left(N_{2}-N_{1}+1, \frac{1}{|\sin (\pi a / q)|}\right) .
$$

(ii) If $S \geq 1$ and $(a, q)=1$ then

$$
\sum_{n \leq S} \min \left(\frac{N}{n}, \frac{1}{|\sin (\pi n a / q)|}\right) \ll\left(\frac{N}{q}+S+q\right) \log (2 q S) .
$$

Put $d n=k$ in the expression for $S_{2}$ to obtain

$$
\begin{aligned}
S_{2} & =\sum_{k \leq u^{2}} \sum_{r \leq N / k}\left(\sum_{d \leq u} \sum_{\substack{n \leq u \\
d n=k}} \mu(d) g(n)\right) e(k r a / q) \\
& \ll(\log x)^{c} \sum_{k \leq u^{2}} \tau(k)\left|\sum_{r \leq N / k} e(k r a / q)\right| .
\end{aligned}
$$

Splitting the $k$-sum according to $\tau(k)>T$ and $\tau(k) \leq T$ and applying Lemma 2 with the choice of $T=(\log x)^{\lambda+4+c}$ yields $S_{2} \ll x / \log ^{\lambda} x$. We write $S_{3}$ as

$$
S_{3}=\sum_{j=0}^{K} \sum_{m \in I_{j}} \tau(m) \sum_{u<n \leq N / m} g(n) e(m n a / q)
$$

where $K$ is defined by $2^{K} u \leq N / u<2^{K+1} u, I_{j}=\left(2^{j} u, 2^{j+1} u\right]$ for each $0 \leq j \leq K-1$ and $I_{K}=\left(2^{K} u, N / u\right]$. Hence

$$
S_{3}=\sum_{j=0}^{K} U_{j}
$$


where, putting $Y_{j}=2^{j} u$ and using the Cauchy-Schwarz inequality, we obtain

$$
\begin{aligned}
\left|U_{j}\right|^{2} & \leq \sum_{m \in I_{j}} \tau^{2}(m) \sum_{m \in I_{j}}\left|\sum_{u<n \leq N / m} g(n) e(m n a / q)\right|^{2} \\
& \ll Y_{j}(\log x)^{2 c+3} \sum_{\substack{n_{1}, n_{2} \\
u<n_{i} \leq N / Y_{j}}}\left|\sum_{\substack{Y_{j}<m \leq 2 Y_{j} \\
m \leq \min \left(N / n_{1}, N / n_{2}\right)}} e\left(m\left(n_{1}-n_{2}\right) a / q\right)\right|
\end{aligned}
$$

which by Lemma 2 yields

$$
\left|U_{j}\right| \ll Y_{j}^{1 / 2} x^{1 / 2}(\log x)^{c+3 / 2}+x /(\log x)^{\lambda+1} .
$$

So finally,

$$
S_{3}=\sum_{j=0}^{K} U_{j} \ll x / \log ^{\lambda} x .
$$

This completes the proof of Theorem 3 .

\section{References}

[1] P. T. Bateman and S. Chowla, Some special trigonometric series related to the distribution of prime numbers, J. London Math. Soc. 38 (1963), 372-374.

[2] A. S. Besicovitch, Problem on continuity, ibid. 36 (1961), 388-392.

[3] H. Davenport, On some infinite series involving arithmetic functions (II), Quart. J. Math. (Oxford) 8 (1937), 313-320.

[4] H. Halberstam and H.-E. Richert, Sieve Methods, Academic Press, London, 1974.

[5] H. Siebert, Einige Analoga zum Satz von Siegel-Walfisz, in: Zahlentheorie (Tagung, Math. Forschungsinst., Oberwolfach, 1970), Bibliographisches Inst., Mannheim, 1971, $173-184$.

[6] R. C. Vaughan, The Hardy-Littlewood Method, 2nd ed., Cambridge Tracts in Math. 125, Cambridge Univ. Press, Cambridge, 1997.

Dipartimento di Matematica Università di Ferrara

Via Machiavelli 35

44100 Ferrara, Italy

E-mail: cod@dns.unife.it
Department of Mathematics University of Glasgow Glasgow G12 8QW, U.K. E-mail:m.nair@maths.gla.ac.uk 